\title{
A Novel Pigeon-Inspired Optimized RBF Model for Parallel Battery Branch Forecasting
}

\author{
Yanhui Zhang $\mathbb{D}^{1,2,3,4}$ Shili Lin ${ }^{1}{ }^{5}$ Haiping Ma ${ }^{5}{ }^{6}$ Yuanjun Guo $\mathbb{D}^{1},{ }^{1,2,3,4}$ \\ and Wei Feng (iD) $1,2,3,4,7,8$ \\ ${ }^{1}$ CAS Key Laboratory of Human-Machine Intelligence-Synergy Systems, Shenzhen Institutes of Advanced Technology, \\ Shenzhen, China \\ ${ }^{2}$ Guangdong Provincial Key Lab of Robotics and Intelligent System, Shenzhen Institutes of Advanced Technology, \\ Chinese Academy of Sciences, Beijing, China \\ ${ }^{3}$ Ultrasonic Nondestructive Engineering Technology Research Center of Guangdong, Shenzhen 518055, China \\ ${ }^{4}$ Shenzhen Institutes of Advanced Technology, Chinese Academy of Sciences, Shenzhen 518055, China \\ ${ }^{5}$ Guangzhou Institute of Energy Conversion, Chinese Academy of Sciences, Guangzhou 510640, China \\ ${ }^{6}$ Department of Electrical Engineering, Shaoxing University, Shaoxing 312000, China \\ ${ }^{7}$ University of Chinese Academy of Sciences, Beijing 100000, China \\ ${ }^{8}$ The Department of Mechanical and Automation Engineering, The Chinese University of Hong Kong, Shatin, Hong Kong
}

Correspondence should be addressed to Yuanjun Guo; yj.guo@siat.ac.cn and Wei Feng; wei.feng@siat.ac.cn

Received 7 August 2020; Revised 20 September 2020; Accepted 10 February 2021; Published 23 February 2021

Academic Editor: Jing $\mathrm{Na}$

Copyright (C) 2021 Yanhui Zhang et al. This is an open access article distributed under the Creative Commons Attribution License, which permits unrestricted use, distribution, and reproduction in any medium, provided the original work is properly cited.

Battery energy storage is the pivotal project of renewable energy systems reform and an effective regulator of energy flow. Parallel battery packs can effectively increase the capacity of battery modules. However, the power loss caused by the uncertainty of parallel battery branch current poses severe challenge to the economy and safety of electric vehicles. Accuracy of battery branch current prediction is needed to improve the parallel connection. This paper proposes a radial basis function neural network model based on the pigeon-inspired optimization method and successfully applies the algorithm to predict the parallel branch current of the battery pack. Numerical results demonstrate the high accuracy of the proposed pigeon-inspired optimized RBF model for parallel battery branch forecasting and provide a useful tool for the prediction of parallel branch currents of battery packs.

\section{Introduction}

As an important part of electric vehicles, the life and safety of lithium-ion battery have a profound impact on the service range of electric vehicles. In order to meet the high power and high energy output requirements of these devices, it is usually necessary to perform series and/or parallel topology on battery modules $[1,2]$. The series connection of batteries can increase the operating voltage, while the parallel connection of batteries can increase the capacity. For seriesconnected battery packs, the battery management system usually provides a balancing module. For parallel battery packs, the single cells will maintain the same terminal voltage and have a certain self-balance. However, the unbalanced current caused by the inconsistency between the single cells will affect the state-of-charge of the battery and the life and safety of the battery pack in the long run [3].

For battery producers, introduction of fully automated production lines does not mean high quality and stable production; in addition to good sources of materials, the management of automated production line is more critical. The inconsistency of the branch batteries in the parallel battery packs will cause current imbalance and reduce battery energy efficiency and even cause some batteries to be overcharged and/or overdischarged.

Battery engineering research mainly focuses on state estimation and how to reduce battery inconsistency for higher energy conversion efficiency and longer battery life, 
while lacking a comprehensive analysis of the battery inconsistency generation mechanism $[4,5]$. The battery management system cannot control the current distribution and energy flow between batteries in parallel modules at this stage, which is directly affected by the battery connection topology. Therefore, it is difficult to implement the control strategy for the inconsistency of parallel batteries in the vehicle application.

In parallel topology, uneven current exists and degradation rates are different between individual batteries. Previous studies on parallel connection [6] focused on several aspects, including the evolution of current distribution under certain conditions, unbalances on the degradation rate of the battery, design and control of balanced circuits, the effects of connection wires and welding techniques [7-10], predicting future capacities, and remaining useful life $[11,12]$.

In the field of battery modelling technology, the current distribution is calculated by numerical simulation. The voltage of various batteries can be extracted from the state of charge-voltage curve under the discharge process. This method considers the influence of internal resistance on battery current, without building any model, but it is necessary to repeatedly search for the voltage of various battery under each time step. Brand et al. [13] explore the current distribution within two parallel-connected batteries, and yet how to extend this method to more than two units has not been discussed. Miyatake examines the relationship between discharge capacities of a multicell battery with two different types, but its topology is unraveled [14]. In addition, they derive a mathematical model for discharge characteristics of the multicell battery based on the equivalent circuit model of an individual battery. Bruen and Marco [15] adopt the modelling and experimental method to the evaluation of parallel-connected lithium-ion batteries for an electric vehicle.

The rest of this paper is organized as follows. Section 2 introduces the preliminaries of radial basis function neural network and pigeon-inspired optimization. Section 3 presents the pigeon-inspired optimization optimized radial basis function (PIO-RBF) neural network. Section 4 shows the experimental setup. Section 5 analyzes branch current forecasting case study, followed by the main findings and future prospects of this work in Section 6 .

\section{Preliminaries}

2.1. Radial Basis Function Neural Network. RBF neural network is a three-layer feedforward analysis network, which is composed of input layer, hidden layer, and output layer. Figure 1 shows the topology of RBF neural network, in which the function of controlling node transformation in hidden layer is radial basis function.

If the input layer of the RBF neural network has $n$ information source nodes, the input layer can be represented as $\left[x_{1}, x_{2}, \ldots, x_{n}\right]^{\mathrm{T}}$. In the hidden layer, Gaussian function is selected as radial basis function, which is expressed as

$$
\phi x-c_{i}=\exp \left(-\frac{\left\|x-c_{i}\right\|^{2}}{2 \sigma_{i}^{2}}\right),
$$

where $\|\cdot\|$ is Euclidean norm, $c_{i}$ is the base function center of the $i$-th node of the hidden layer, $c$ is the width of the basis function of the $i$-th node in the layer, and $\sigma_{i}$ is the width of the basis function of the $i$-th node in the layer.

The output layer of the RBF neural network can be obtained by linearly weighting the hidden layer nodes. If the output layer has $k$ output nodes, the $j$-th output of the RBF neural network can be expressed as

$$
y_{i}=\sum_{i=1}^{k} w_{i j} \exp \left(-\frac{x-c_{i}^{2}}{2 \sigma_{i}^{2}}\right) .
$$

In the above formula, $w$ is the connection weight from the $i$-th hidden layer node to the $j$-th output layer; $Y$ is the node value of the $j$-th output layer.

The number determining the hidden layer nodes is also the key to realize RBF neural network, in addition to training and optimizing the center $C_{i}$ of hidden layer basis function, the width of hidden layer node $\sigma_{i}$, and the weight factor $W$ from hidden layer to output layer. Theoretically, with the number of hidden layer nodes increasing, the approximation ability of RBF neural network is stronger and the output is more accurate. However, in practical application, the increase of hidden layer nodes will lead to the increase of training time and learning cost, and the generalization ability of the network will decrease, which will lead to the overlearning and the fault tolerance ability of neural network.

In order to determine the optimal number of hidden layer nodes of RBF neural network, this paper introduces the trial-and-error method. First obtain the initial interval $[a, b]$ of the number of hidden layer nodes of the RBF neural network through the empirical formula, and then calculate and compare the points in the interval one by one to narrow the interval of the number of nodes to determine the optimal number of hidden layer nodes.

$$
a=\frac{n_{0}+n_{i}}{2} \leq n_{h} \leq\left(n_{i}+n_{0}\right)+10=b,
$$

where $n_{i}, n_{h}$, and $n_{0}$ are the number of nodes in the input layer, hidden layer, and output layer of the RBF neural network, respectively.

2.2. Pigeon-Inspired Optimization. When the pigeon is far away from the destination, it arrives at the destination with the help of the geomagnetic field and landmark buildings. The key reasons that affect the homing of pigeons can be divided into three categories. The first reason is the sun, the second reason is the Earth's magnetic field, and the third reason is the geomorphic landscape. During the flight of the pigeons, different cruise tools will be used according to situations. In order to idealize some of the homing characteristics of pigeons, two operators are designed by using some rules. 


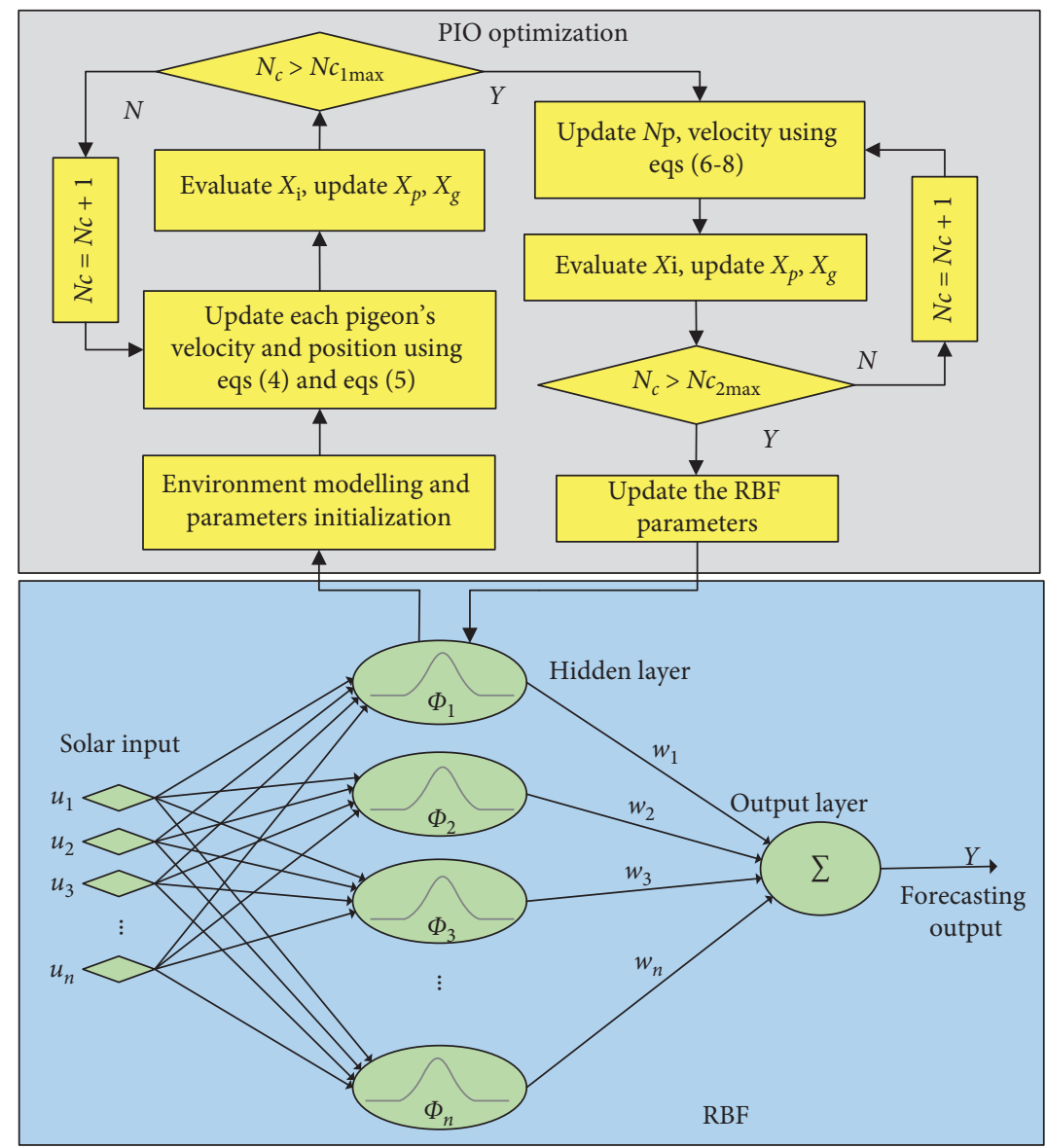

FIgUre 1: Procedure of PIO-optimized RBF network.

(1) Map and Compass Operator. Pigeons can sense the Earth field by using magnetoreception to shape the map in their brains. They regard the altitude of the sun as compass to adjust the direction. As they fly to their destination, they rely less on sun and magnetic particles.

(2) Landmark Operator. When the pigeons fly close to their destination, they will rely on landmarks neighboring them. If they are familiar with the landmarks, they will fly straight to the destination. If they are far from the destination and unfamiliar with the landmarks, they will follow the pigeons who are familiar with the landmarks.

The map model is based on the geomagnetic field. $x_{i}$ and $v_{i}$ represent the position and velocity of the $i$-th pigeon, respectively. With the number of iterations increasing, the pigeon constantly learns from the optimal individual within the interaction range and updates its speed $V_{i}$ and position $X_{i}$ in real time:

$$
\begin{aligned}
& V_{i} t=V_{i} t^{*} e^{-r t}+\operatorname{rand} X_{g}-X_{i} t-1, \\
& X_{i} t=X_{i} t-1+V_{i}(t) .
\end{aligned}
$$

The speed of the $i$-th pigeon is determined by the speed of the previous generation and the current best position of the pigeon. $R$ is a map factor, rand is a random number, and $t$ is an algebra; the position of the $i$-th pigeon is determined by its previous position and current speed. The flight of all pigeons is ensured by the map, and then we can get the best position of the pigeons $X g$. Various pigeons will adjust its direction and fly to the pigeon with the best position according to (4), while (5) will adjust its position.

The landmark model is built with pigeons using landmarks for navigation. When using landmarks to navigate, the distance from the destination is closer than that from map navigation. If the pigeon is not familiar with the current location landmark, it will fly under the guidance of nearby pigeons; when finding landmark buildings or familiar 
Input

$N_{P}$ : number of individuals in pigeon swarm

$D$ : dimension of the search space

$R$ : the map and compass factor

Search range: the borders of the search space

$N c 1_{\text {max }}$ : the maximum number of generations that the map and compass operation is carried out

$N c 2_{\text {max }}$ : the maximum number of generations that the landmark operation is carried out.

Output

$\mathrm{Xg}$ : the global optima of the fitness function $\mathrm{f}$

(1) Initialization

Set initial values for $N c 1_{\max }, N c 2_{\max }, N P, D, R$ and the search range

Set initial path $X i$ and velocity $V_{i}$ for each pigeon individual

Set $X_{p}=X_{i}, N C=1$

Calculate fitness values of different pigeon individuals

$X_{g}:=\operatorname{argmin}\left[f:\left(X_{p}\right)\right]$

(2) Map and compass operations

For $N c=1$ to $N c 1_{\max }$ do

for $i=1$ to $N p$ do

while $X_{i}$ is beyond the search range do

calculate $V_{i}$ and $X_{i}$ according to equations (4) and (5)

end while

end for

evaluate $X_{i}$, and update $X_{p}$ and $\mathrm{Xg}$

end for

(3) Landmark operations

For $N c=N c 1_{\text {max }}+1$ to $N c 2_{\text {max }}$ do

while $X_{p}$ is beyond the search range do

rank all the available pigeon individuals according to their fitness values

$N P=N P / 2$

keep half of the individuals with better fitness value, and abandon the other half

$X_{c}=$ average value of the paths of the remaining pigeon individuals

calculate $X_{i}$ according to equation (8)

end while

evaluate $X_{i}$, and update $X_{p}$ and $X_{g}$

end for

(4) Output

$X_{g}$ is output as the global optima of the fitness function $f$

Algorithm 1: PIO algorithm.

locations, it will fly freely according to experience. In the landmark model, $N_{p}$ is used to record the number of half pigeons in each generation, and $X_{c}(t)$ is the central position of all pigeons in generation $t$. If various pigeon can fly a straight distance to the destination, the following formula will be obtained:

$$
\begin{aligned}
& N_{P}(t)=\frac{N_{p}(t-1)}{2}, \\
& X_{c}(t)=\frac{\sum X_{i}(t)^{*} \text { fitness } X_{i}(t)}{N_{p} \sum \text { fitness } X_{i}(t)}, \\
& X_{i}(t)=X_{i}(t-1)+\operatorname{rand}^{*}\left(X_{c}(t)-X_{i}(t-1)\right),
\end{aligned}
$$

$$
\text { fitness }\left(X_{i}(t)\right)=\frac{1}{f_{\min }\left(X_{i}(t)\right)} \text {. }
$$

In the above equation, fitness $(X)$ is the mass of pigeon, equation (9) is for the minimum optimization problem, and equation (10) is for the maximum optimization problem.

\section{Proposed PIO Optimized RBF Neural Network}

In this paper, pigeon-inspired optimization is adopted where PIO method is introduced in training the linear and nonlinear parameters given a predetermined RBF network model structure. The detailed implementation procedure of 


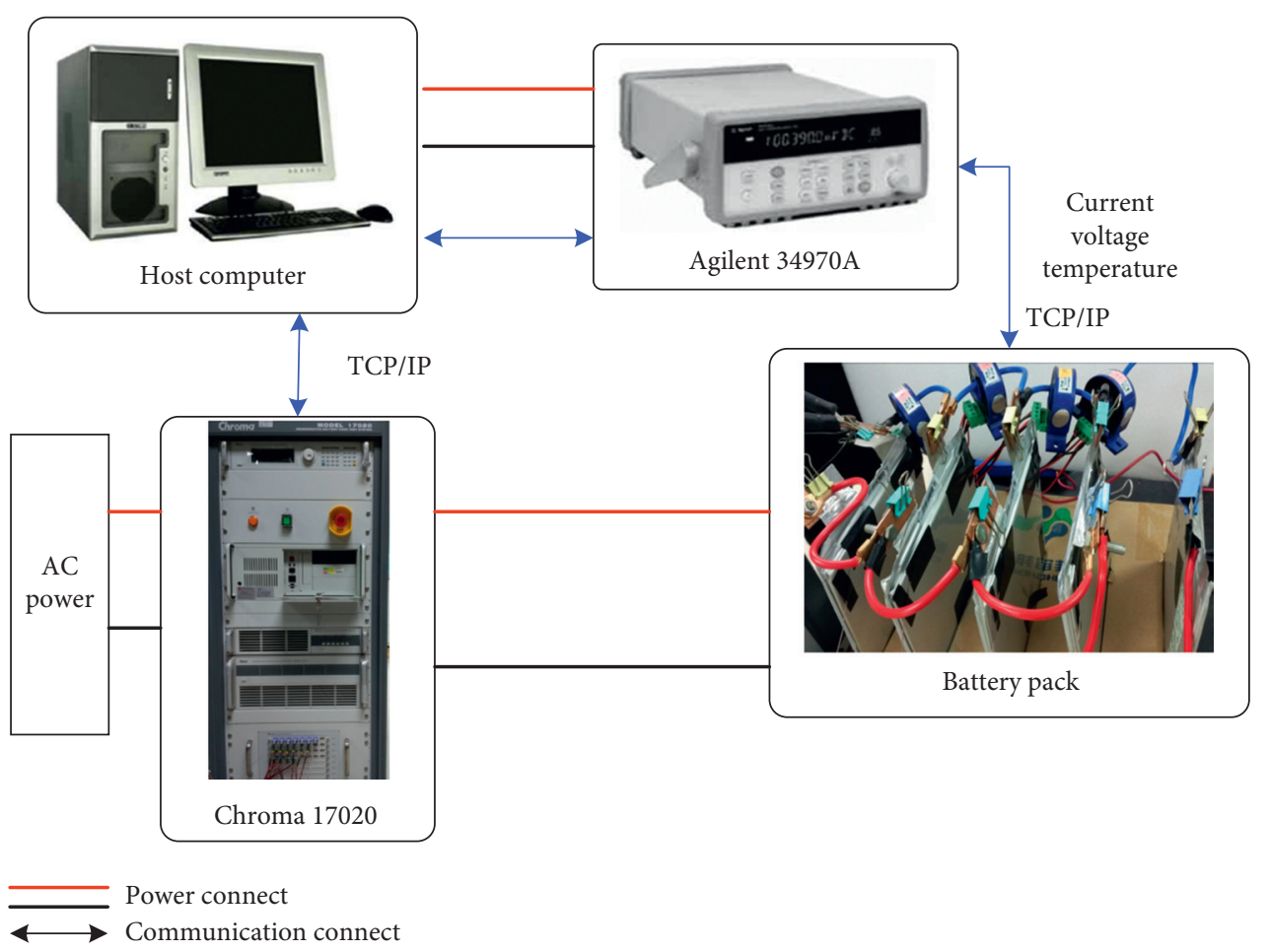

FiguRE 2: Experimental setup for implementation of the parallel-connected batteries.

PIO for current branch path forecasting can be described as follows (as shown in Figure 1) (Algorithm 1).

\section{Experiment}

4.1. Experimental Battery. The lithium-ion battery used in the research is LiFePO4/graphite battery. In the experiment, LiFePO4 battery with a nominal voltage of $3.65 \mathrm{~V}$ and nominal capacity of $20 \mathrm{Ah}$ was selected, and its size (thickness $\times$ width $\times$ length) was $52 \mathrm{~mm} \times 64 \mathrm{~mm} \times 102 \mathrm{~mm}$. The positive electrode of the battery is the thickness of aluminum foil of $15-25 \mu \pi \iota$ and anode foil thickness of $12-20 \mu \pi \iota$; negative electrode is made of nickel-plated copper, of $0.2-0.3 \mathrm{~mm}$ thickness and $20-60 \mathrm{~mm}$ width; plastic film is with a thickness of $0.153 \mathrm{~mm}$. Polyvinylidene fluoride (PVDF) and N-methyl-2-pyrrolidone (NMP) were used as binder in manufacturing of both electrodes. The electrolyte consisted of $1 \mathrm{MLiPF}_{6}$ in ethylene carbonate (EC)-dimethyl carbonate (DMC)-ethyl methyl carbonate (EMC) $(1: 1: 1$, mass ratio) electrolyte.

4.2. Experimental System. To explore the current distribution and overall characteristics of parallel battery pack under different discharge processes, an experimental system of $\mathrm{LiFePO}_{4}$ /graphite battery parallel battery pack was developed. The test bench is composed of battery pack, test system, data acquisition system, and management system machine. The charging and discharging programming software is built in the management system. In Figure 2, an experimental device for parallel battery test is shown. The data acquisition system (Agilent 34970A, Agilent Limited) can measure voltage with a resolution of 6.5 bits, and the measurement accuracy can reach $0.004 \%$. It is applied to voltage measurement to improve the sample precision of voltages. The measurement system of parallel battery pack is carried out at normal temperature. The charge and discharge management of battery pack is carried out with 17020 programmable measurement system. The abovementioned test system provides independent channels to support the charge and discharge experiment of multiple battery modules.

The Hall current sensor is the key to accurately measure the current distribution in parallel battery packs. Ordinary current shunts are accurate in measuring string current, but in most cases, the increased resistance will seriously affect the current distribution. This is why the contactless current sensor is more suitable for measuring the current distribution of parallel batteries in this work.

In order to measure the current distribution of parallel battery packs, the LHB50A5VY2 Hall-effect current sensor is used in the parallel circuit of the test battery pack. The accuracy of the Hall current sensor is $\pm 0.5 \%$, and the temperature drift coefficient is $\pm 0.5 \mathrm{mv} /{ }^{\circ} \mathrm{C}$. The Hall current sensor can accurately obtain the current through the wire, and the actual current through the battery is equal to the difference between the values of adjacent Hall current sensors.

The Hall current sensor does not need to be connected to the parallel loop, eliminating the influence of the shunt resistance. In this project, the constant current discharge test was mainly carried out to explore the current distribution condition of the parallel battery branch, and the RBF network optimized by the pigeon group is used to predict the current of the parallel branch. 


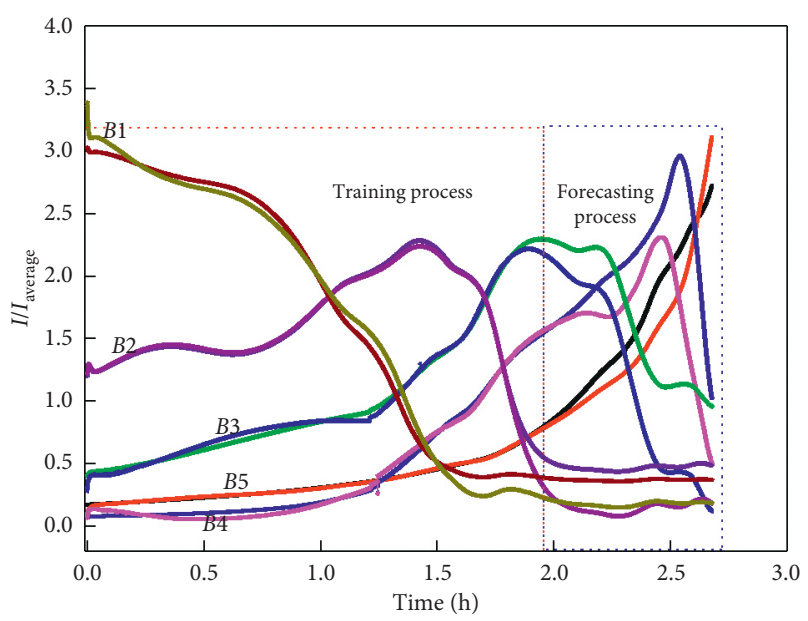

$$
\begin{aligned}
& \text {-.- - B5 training + forecasting ... } \quad B 4 \text { training + forecasting } \\
& \text {.. - } B 3 \text { training + forecasting ... } \quad B 2 \text { training + forecasting } \\
& \text {.. - B1 training + forecasting _... B4-truth } \\
& \text {... - B5-truth .... - B2-truth } \\
& \text {-.*- B3-truth }
\end{aligned}
$$

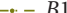

FIGURE 3: Actual and forecasting current scenario of parallel branch.

\section{Branch Current Forecasting Case Study}

In this section, the competitive performance of the proposed PIO optimized RBF neural network is adopted in forecasting real world current branches in parallel battery packs scenario. The data is collected from the abovementioned experiment where the batteries are placed under a normal laboratory environment. The data recorded every 1 second ranging from first branch to fifth branch under the $0.3 \mathrm{C}$ discharge rate are adopted in the modelling. In view of the sensors investment, branch current prediction can effectively reduce the number of current sensors in engineering practice.

The research on current prediction and prediction accuracy has always been a topic of concern to researchers due to the demand of predicting the branch current distribution characteristics of parallel batteries. In this paper, based on the analysis of the current distribution uncertainty characteristics of parallel battery pack, the pigeon-inspired optimization algorithm is combined with RBF neural network to explore the parallel branch current forecasting. The actual and prediction current scenarios of parallel branch are shown in Figure 3; and the errors of current scenario are shown in Figure 4.

It could be clearly observed in Figure 4 that the PIO achieved a comparatively accurate forecasting curve on training stage possibly due to the parameter training efforts, and yet the error in the prediction stage can be further reduced in the future. The reason for larger error of battery close to load is mainly due to the large change of curve when approaching the battery cutoff voltage.

By comparison of performance of battery module in

\begin{tabular}{|c|c|c|c|}
\hline $\begin{array}{l}\text { Points of } \\
\text { comparison }\end{array}$ & $\begin{array}{c}\text { The battery } \\
\text { farthest from } \\
\text { the load }(B 5) \\
(\%)\end{array}$ & $\begin{array}{l}\text { Battery in } \\
\text { the middle } \\
(B 3)(\%)\end{array}$ & $\begin{array}{c}\text { Battery } \\
\text { closest to the } \\
\text { load }(B 1) \\
(\%)\end{array}$ \\
\hline \multicolumn{4}{|l|}{ Training } \\
\hline $\begin{array}{l}\text { The error ratio of } \\
\text { branch current at the } \\
\text { beginning }\end{array}$ & 26.15 & 11.8 & 10.1 \\
\hline $\begin{array}{l}\text { The error ratio of } \\
\text { branch current at the } \\
\text { end of discharging }\end{array}$ & 1.22 & 1.89 & 4.89 \\
\hline $\begin{array}{l}\text { The maximum error } \\
\text { ratio of branch } \\
\text { current at the } \\
\text { discharging }\end{array}$ & 41.45 & 11.8 & 30.5 \\
\hline $\begin{array}{l}\text { The average error } \\
\text { ratio of branch } \\
\text { current at the } \\
\text { discharging }\end{array}$ & 1.69 & 1.56 & 5.7 \\
\hline Standard error & 3.07 & 1.16 & 7.1 \\
\hline \multicolumn{4}{|l|}{ Forecasting } \\
\hline $\begin{array}{l}\text { The error ratio of } \\
\text { branch current at the } \\
\text { beginning }\end{array}$ & 1.20 & 1.90 & 4.91 \\
\hline $\begin{array}{l}\text { The error ratio of } \\
\text { branch current at the } \\
\text { end of discharging }\end{array}$ & 12.6 & 35.1 & 1.2 \\
\hline $\begin{array}{l}\text { The maximum error } \\
\text { ratio of branch } \\
\text { current at the } \\
\text { discharging }\end{array}$ & 22.6 & 35.1 & 5.9 \\
\hline $\begin{array}{l}\text { The average error } \\
\text { ratio of branch } \\
\text { current at the } \\
\text { discharging }\end{array}$ & 12.24 & 14.0 & 2.2 \\
\hline Standard error & 6.54 & 9.9 & 1.7 \\
\hline
\end{tabular}
parallel, the prediction research of parallel branch current

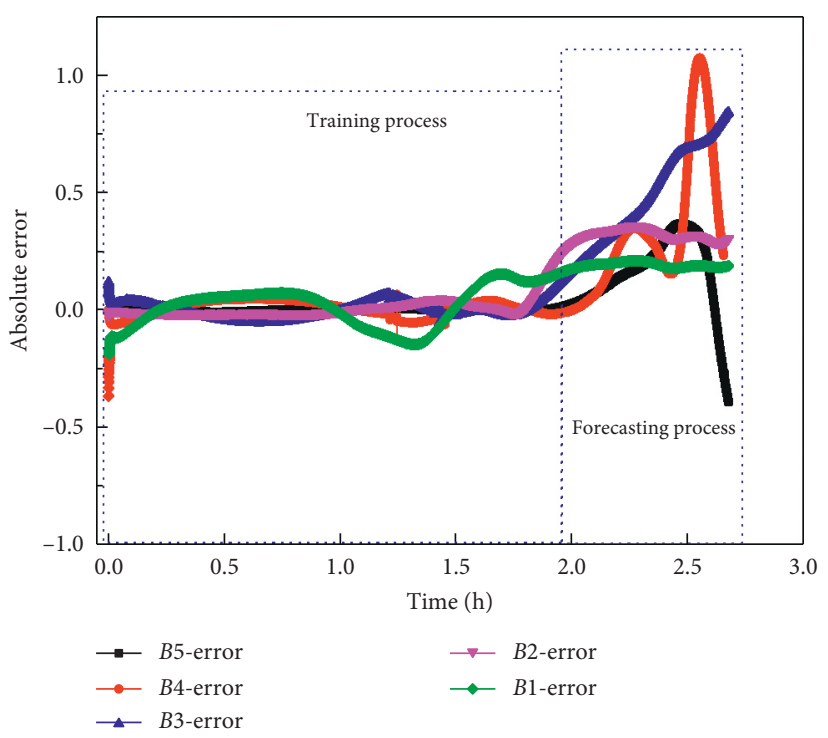

Figure 4: Actual and forecasting error of current scenario.

TABLE 1: Comparison of performance of parallel battery module. 
based on PIO optimized RBF was explored, focusing on the maximum error and standard deviation of various parallel branches in the training and prediction stage. It can be clearly found that the standard error and the average error ratio of branch current in the middle position of the parallel battery pack are relatively better than $B 5$ and $B 1$, which may be due to the fact that the current variation of the battery is not very sharp, as shown in Table 1.

Moreover, the average error and standard deviation of the battery close to the load end are in the same level as those of the battery far away from the load, but the maximum error ratio of branch current is relatively large in the training stage.

\section{Conclusion and Future Work}

A radial basis function (RBF) neural network based on pigeon colony optimization is proposed to predict the current distribution of parallel batteries. The model structure can optimize the parameters of RBF neural network. The competitive performance of the model has been verified in the current branch prediction task of parallel battery pack. The experimental and prediction results are proved to be effective, which provides a new idea for reducing the investment of current detection sensor in parallel battery pack.

The future work may be to further optimize the pigeon colony algorithm, further improve the prediction accuracy, and optimize the proposed PIO-RBF model. In addition to its application in the branch current prediction of parallel batteries, the proposed PIO-RBF network has the potential to predict power demand, to forecast wind power generation and many other various random nonlinear power and energy tasks, which offers many possible approaches to shape the future of low-carbon energy.

\section{Data Availability}

Research data are mainly from the experiment in this manuscript.

\section{Conflicts of Interest}

The authors declare that they have no conflicts of interest.

\section{Acknowledgments}

The authors are grateful to the financial support from Guangdong-Hong Kong-Macao Joint Laboratory of HumanMachine Intelligence-Synergy Systems (\#2019B121205007), the Science and Technology Innovation Commission of Shenzhen (No. ZDSYS20190902093209795), National Natural Science Foundation of China (No. U1813222), the National Natural Science Foundation of Guangdong (No. 2016A030313177), Guangdong Frontier and Key Technological Innovation (No. 2017B090910013), the Science and Technology Innovation Commission of Shenzhen (Nos. JCYJ20170818153048647, JCYJ20180507182239617, and JCYJ2018050718223961), and
Zhejiang Provincial Natural Science Foundation of China under Grant No. LY19F030011.

\section{References}

[1] M. Latifi, A. Rastegarnia, A. Khalili, and S. Sanei, "Agent-based decentralized optimal charging strategy for plug-in electric vehicles," IEEE Transactions on Industrial Electronics, vol. 66, no. 5, pp. 3668-3680, 2019.

[2] A. Hübler and O. Wu, "Digital quantum batteries: energy and information storage in nanovacuum tube arrays," Complexity, vol. 15, pp. 48-55, 2010.

[3] R. Xiong, Y. Duan, J. Cao, and Q. Yu, "Battery and ultracapacitor in-the-loop approach to validate a real-time power management method for an all-climate electric vehicle," Applied Energy, vol. 217, pp. 153-165, 2018.

[4] G. Kirbis, D. Selcan, M. Kos, and I. Kramberger, "High performance autonomous charge equalization in series connected batteries," IEEE Transactions on Aerospace and Electronic Systems, vol. 55, no. 1, pp. 95-107, 2019.

[5] M. Dehmer, X. Li, and Y. Shi, "Connections between generalized graph entropies and graph energy," Complexity, vol. 21, no. 1, pp. 35-41, 2015.

[6] M.-S. Wu, C.-Y. Lin, Y.-Y. Wang, C.-C. Wan, and C. R. Yang, "Numerical simulation for the discharge behaviors of batteries in series and/or parallel-connected battery pack," Electrochimica Acta, vol. 52, no. 3, pp. 1349-1357, 2006.

[7] M. Dubarry, A. Devie, and B. Y. Liaw, "Cell-balancing currents in parallel strings of a battery system," Journal of Power Sources, vol. 321, pp. 36-46, 2016.

[8] W. Shi, X. Hu, C. Jin, J. Jiang, Y. Zhang, and T. Yip, "Effects of imbalanced currents on large-format LiFePO 4/graphite batteries systems connected in parallel," Journal of Power Sources, vol. 313, pp. 198-204, 2016.

[9] T. Grün, K. Stella, and O. Wollersheim, "Influence of circuit design on load distribution and performance of parallel-connected Lithium ion cells for photovoltaic home storage systems," Journal of Energy Storage, vol. 17, pp. 367-382, 2018.

[10] M. P. Aghababa, "Fractional modeling and control of a complex nonlinear energy supply-demand system," Complexity, vol. 20, no. 6, pp. 74-86, 2015.

[11] K. Liu, Y. Shang, Q. Ouyang, and W. D. Widanage, “A data-driven approach with uncertainty quantification for predicting future capacities and remaining useful life of lithium-ion battery," IEEE Transactions on Industrial Electronics, vol. 68, no. 4, p. 3170, 2021.

[12] K. Liu, X. Hu, Z. Wei, Y. Li, and Y. Jiang, "Modified Gaussian process regression models for cyclic capacity prediction of lithium-ion batteries," IEEE Transactions on Transportation Electrification, vol. 5, no. 4, pp. 1225-1236, 2019.

[13] M. J. Brand, M. H. Hofmann, M. Steinhardt, S. F. Schuster, and A. Jossen, "Current distribution within parallel-connected battery cells," Journal of Power Sources, vol. 334, pp. 202-212, 2016.

[14] S. Miyatake, Y. Susuki, T. Hikihara, S. Itoh, and K. Tanaka, "Discharge characteristics of multicell lithium-ion battery with nonuniform cells," Journal of Power Sources, vol. 241, pp. 736-743, 2013.

[15] T. Bruen and J. Marco, "Modelling and experimental evaluation of parallel connected lithium ion cells for an electric vehicle battery system," Journal of Power Sources, vol. 310, pp. 91-101, 2016. 